\title{
Global Existence of Small Solutions to Semilinear Schrödinger Equations with Gauge Invariance
}

By

\author{
Hiroyuki CHIHARA*
}

\begin{abstract}
We present the global existence theorem for semilinear Schrödinger equations satisfying gauge invariance. Combining the local existence results and a priori estimates, we construct global solutions with small initial data.
\end{abstract}

\section{§1. Introduction}

In this paper we study the initial value problem for semilinear Schrödinger equations

$$
\begin{gathered}
\partial_{t} u-i \Delta u=F(u, \nabla u), \quad \text { in }(0, \infty) \times \mathbf{R}^{N}, \\
u(0, x)=u_{0}(x), \quad \text { in } \mathbf{R}^{N},
\end{gathered}
$$

where $u(t, x)$ is complex-valued, $i=\sqrt{-1}, \partial_{t}=\partial / \partial t, \partial_{x}=\partial / \partial_{x}, \quad(j=1, \cdots, N), \Delta$ $=\sum_{j=1}^{N} \partial_{x}^{2}$, and $N \in \mathbf{N}$. We assume that the nonlinear term $F(u, q): \mathbf{C} \times \mathbf{C}^{N} \rightarrow \mathbf{C}$ satisfies

$$
\begin{gathered}
F(u, q) \in C^{\infty}\left(\mathbf{R}^{2} \times \mathbf{R}^{2 N}\right), \\
|F(u, q)| \leqslant C\left(|u|^{p w}+|q|^{p w}\right), \quad \text { near }(u, q)=0, \quad p_{w}=2,3, \cdots .
\end{gathered}
$$

We see the second variable $q$ corresponding to $\nabla u=\left(\partial_{x_{1}} u, \cdots, \partial_{x_{N}} u\right)$. We define

Communicated by T. Kawai, March 4, 1994. Revised May 16, 1994.

1991 Mathematics Subject Classifications: 35G25, 35Q55

* Department of Applied Mathematics and Physics, Kyoto University, Kyoto 606-01, Japan.

Current address: Department of Mathematical Sciences, Shinshu University, Matsumoto 390, Japan. 


$$
\begin{gathered}
\frac{\partial}{\partial u}=\frac{1}{2}\left(\frac{\partial}{\partial v}-i \frac{\partial}{\partial w}\right), \quad \frac{\partial}{\partial \bar{u}}=\frac{1}{2}\left(\frac{\partial}{\partial v}+i \frac{\partial}{\partial w}\right), \\
\frac{\partial}{\partial q_{j}}=\frac{1}{2}\left(\frac{\partial}{\partial \xi_{j}}-i \frac{\partial}{\partial \eta_{j}}\right), \quad \frac{\partial}{\partial \bar{q}_{j}}=\frac{1}{2}\left(\frac{\partial}{\partial \xi_{j}}+i \frac{\partial}{\partial \eta_{j}}\right), \\
v=\operatorname{Re} u, \quad w=\operatorname{Im} u, \quad \xi_{j}=\operatorname{Re} q_{j}, \quad \eta_{j}=\operatorname{Im} q_{j}, \quad j=1, \cdots, N .
\end{gathered}
$$

The purpose of this paper is to prove the global existence of small solutions to (1.1)-(1.2). Generally, Schrödinger type equations have so-called loss of derivatives because of the first order terms and therefore we cannot obtain classical energy estimates. More precisely, the classical energy estimates hold if and only if

$$
\operatorname{Im} \frac{\partial F}{\partial q_{j}}(u, q) \equiv 0, \quad j=1, \cdots, N
$$

It was difficult even to prove the local existence unless (1.5) holds. But recently, several results on the local existence appeared without the condition (1.5) (see A. Soyeur [15] , C. E. Keing-G. Ponce-L. Vega [9] N. Hayashi-T. Ozawa [5] and H. Chihara [1] [2]). Then studies on the global existence have been mainly concerned with the case (1.5) or the special cases without (1.5). These results are the following.

(1) Assume (1.5). If $N\left(p_{w}-1\right)^{2} / 2 p_{w}>1$, namely

$$
N \geqslant \begin{cases}5, & \left(p_{w}=2\right), \\ 2, & \left(p_{w}=3\right), \\ 1, & \left(p_{w}=4\right),\end{cases}
$$

then (1.1) - (1.2) has a global solution with small initial data (see S. Klainerman-G. Ponce [10] and J. Shatah [14]). They established so-called $L^{p}-$ $L^{q}$ method and applied to several nonlinear equations. N. Hayashi [4] studied some quadratic nonlinear equations in the case of $N=3,4$. Using some operators which have good commutation relations to $e^{i \Delta t}$, he proved global existence results (see also T. Ozawa [13, Theorem 1]).

(2) If $N=1$, the problem (1.1)-( 1.2$)$ is easier than the case of $N \geqslant 2$, because a gauge transform

$$
u(t, x) \mapsto v(t, x) \equiv u(t, x) \exp \left(-\frac{1}{2} \int_{-\infty}^{x} \operatorname{Im} \frac{\partial F}{\partial q}\left(u, \partial_{x} u\right)(t, y) d y\right)
$$

eliminates the bad first order term $\operatorname{Im}(\partial F / \partial q) \partial_{x} u$ (see N. Hayashi-T. Ozawa [5] and H. Chihara [1]). Recently, S. Katayama-Y. Tsutsumi [8] studied the global existence of small solutions without (1.5). If $p_{w}=3, F$ is gauge invariant 
(see (1.7)) and the cubic term of $F$ satisfies "null gauge condition" which was introduced by Y. Tsutsumi [17], then the global existence results hold. Even if we assume (1.5) additionally, we can see their results as some extension of [10] [14] (see also Y. Tsutsumi [17]).

(3) If the nonlinear term satisfies

$$
\partial_{x_{k}}\left(\operatorname{Im} \frac{\partial F}{\partial q_{j}}(u, \nabla u)\right)-\partial_{x},\left(\operatorname{Im} \frac{\partial F}{\partial q_{k}}(u, \nabla u)\right)=0
$$

for any $u \in C^{1}\left(\mathbf{R}^{N}\right)$ and $j, k=1, \cdots, N$, which is equivalent to the condition that there exists a real-valued function $G(u)$ such that

$$
\partial_{x, G}(u)=\operatorname{Im} \frac{\partial F}{\partial q_{j}}(u, \nabla u)
$$

for any $u \in C^{1}\left(\mathbf{R}^{N}\right)$ and $j=1, \cdots, N$, then a gauge transform

$$
u(t, x) \mapsto v(t, x) \equiv u(t, x) \exp \left(-\frac{1}{2} G(u(t, x))\right)
$$

is available similarly to the case of $N=1$. Under this assumption, it is easy to see that analogous results to [10] [14] hold. This fact appeared basically in A. Soyeur's work on Ishimori equations [15]. T. Ozawa [13] also studied the global existence for some quadratic semilinear equations with this type of nonlinearity.

(4) Recently, N. Hayashi-T. Ozawa [6] studied radially symmetric solutions. In this case, the difficulty coming from the loss of derivatives reduces to that of one dimensional case. Then they proved the global existence of small solutions.

The above-mentioned works, except for [2] [9], delt with the case of (1.5) or the case in which the gauge transform is available. C. E. Kenig-G. Ponce-L. Vega [9] succeeded in getting local solutions to general semilinear equations with small initial data. Analyzing local smoothing property of $e^{i \Delta t}$, they constructed the inverse of Schrödinger type operators essentially. More precisely, they proved that the operators $\partial_{x} /\left(\partial_{t}-i \Delta\right), j=1, \cdots, N$ were bounded in some sense. To get the inverse of Schrödinger type operators by Neumann series, the coefficients of the first order terms must be small. In semilinear equations, these coefficients are functions of solution. Therefore the smallness of solutions is essential for their method. It seems to be defficult to extend their results to global existence. On the other hand, S. Doi [3] established a rather simple and useful method for linear Schrödinger type equations. Actually, in [2], using his method with some modifications, we proved the local existence theorem for general semilinear equations with large initial data. In this paper we will con- 
struct a global solution to $(1.1)-(1.2)$ with this local existence results and $a$ priori estimates. Our main results are the following.

Theorem 1.1. Let $N \geqslant 3$ and let $m$ be a sufficiently large integer. Assume that $F$ satisfies (1.3), (1.4) with $p_{w}=3$ and gange invariance

$$
F\left(e^{i \theta} u, e^{i \theta} q\right)=e^{i \theta} F(u, q), \quad \text { for }(u, q) \in \mathbf{C} \times \mathbf{C}^{N}, \theta \in \mathbb{R} .
$$

Then there exists a constant $\varepsilon_{0}>0$ such that if $u_{0} \in \bigcap_{j=0}^{N+1} H^{m+2-j, j}$ satisfies $\left\|u_{0}\right\|_{\substack{N=1 \\ N=1} H^{m-j,}}=\varepsilon \leqslant \varepsilon_{0}$, then the initial value problem $(1.1)-(1.2)$ possesses a unique solution $u \in \bigcap_{j=0}^{N+1} C\left([0, \infty) ; H^{m+2-j, j}\right)$. Here

$$
\begin{gathered}
H^{s, p}=\left\{u \in \mathscr{S}^{\prime}\left(\mathbb{R}^{N}\right) \mid\|u\|_{H^{s, p}}<+\infty\right\}, s, p \in \mathbb{R}, \\
\|u\|_{H^{s, p}}=\left\|\langle x\rangle^{p}\langle D\rangle^{s} u\right\|_{L^{2},},\langle x\rangle=\left(1+|x|^{2}\right)^{1 / 2}, \quad\langle D\rangle=(1-\Delta)^{1 / 2} .
\end{gathered}
$$

$H^{s, 0}=H^{s} . \&$ and $\mathscr{\&}^{\prime}$ denote Schwartz class and its topological dual space respectively.

Remark 1.2. The infinum of the integer $m$ is determined by two factors. One is embedding and we require $[m / 2] \geqslant N+2$. Another is concerned with the properties of pseudo-differential operators and we need $m>N / 2+l^{*}$ with some $l^{*} \in \mathbb{N}$. It may be sufficient provided that $m \geqslant 8 N$.

Our idea of proof consists of local existence and a priori estimates. Concerning the former, let us consider

$$
\partial_{t} u-i \Delta u+\sum_{j=1}^{N} b_{j}(x) \partial_{x, u}+\sum_{j=1}^{N} \beta_{j}(x) \partial_{x} \bar{u}=g(t, x), \quad(N \geqslant 2)
$$

$\operatorname{Im}\left(b_{j}(x)\right) \partial_{x}, u$ gives loss of derivatives and $\beta_{j}(x) \partial_{x}, \bar{u}$ does not. But if we try to use linear theory with some transformation $K$ (see S. Doi [3] and S. Mizohata $[12])$, then we can eliminate $\operatorname{Im}\left(b_{j}(x)\right) \partial_{x}, u$ certainly but another loss of derivatives comes from $\beta_{j}(x) \partial_{x}, \bar{u}$ because the transformation $K$ generally has the property $K \bar{u} \neq \overline{K u}$. To avoid this difficulty, we study $2 \times 2$ systems of $(u, \bar{u})$. Combining diagonalization and S. Doi's method, we can easily get local solutions to $(1.1)-(1.2)$. Concerning a priori estimates, since our method is based on linear theory, it is necessary that $\operatorname{Im}\left(\partial F / \partial q_{j}\right), j=1, \cdots, N$, which are imaginary parts of coefficients of $\partial_{x j} u$, are integrable on any line segment in $\mathbb{R}^{N}$ and these integrals are uniformly bounded (see J. Takeuchi [16] and S. Mizohata [12]). Then we need to introduce weighted Sobolev spaces. But direct estimates in the weighted Sobolev spaces are useless for global existence because a commutator $\left[\langle x\rangle^{p}, \Delta\right]$ gives another linear terms. To avoid this difficulty, we assume gauge 
invariance (1.7). More precisely, we consider the following well-known operators

$$
J_{k}(t) u=e^{i|x|^{2 / 4}(1+t)} 2 i(1+t) \partial_{x_{k}}\left(e^{-i|x|^{2 / 4}(1+t)} u\right)=\left(x_{k}+2 i(1+t) \partial_{x_{k}}\right) u
$$

for $u \in \&\left(\mathbf{R}^{N}\right), k=1, \cdots, N . J={ }^{t}\left(J_{1}, \cdots, J_{N}\right)$ satisfies

$$
\left[\partial_{t}-i \Delta, J_{k}\right]=0, \quad\left[\partial_{x}, J_{k}\right]=\delta_{j k}, \quad j, k=1, \cdots, N
$$

Under the assumption (1.7), $J$ acts on $F$ as if it were the usual differential operator, and then we can use chain-rule with respect to $J$. Hence we can get estimates in the weighted Sobolev spaces without giving another linear terms (see $§ 3)$.

Before the end of this section, we present the organization of this paper. In $\S 2$ we study some linear Schrödinger type systems and obtain a sufficient condition of $L^{2}$-wellposedness. $\S 3$ consists of preliminaries. We give the local existence theorem and consider the relation between the existence time and the regularity of initial data. Besides we prepare properties of nonlinear term and some useful embedding concerned with $J$. Finally in $\S 4$ we will complete the proof of Theorem 1.1.

\section{§2. Some Linear Systems}

In this section we will rewrite the results in $[2, \S 2]$ as a convenient form to apply to the global existence. Let us consider the following linear systems

$$
\begin{gathered}
\left(\partial_{t}+i H(t)\right) v=f(t, x), \quad \text { in } \quad(0, T) \times \mathbf{R}^{N}, \\
v(0, x)=v_{0}(x), \quad \text { in } \mathbf{R}^{N},
\end{gathered}
$$

where $v(t, x)={ }^{t}\left(v_{1}(t, x), v_{2}(t, x)\right)$ is $\mathbf{C}^{2}$-valued, $f(t, x)={ }^{t}\left(f_{1}(t, x), f_{2}(t, x)\right)$, and the operator $H(t)=h(t, x, D)$ is defined by

$$
\begin{gathered}
h(t, x, \xi)=a(\xi)+b(t, x, \xi), \quad a(\xi)=\left[\begin{array}{cc}
|\xi|^{2} & 0 \\
0 & -|\xi|^{2}
\end{array}\right], \\
b(t, x, \xi)=\left[\begin{array}{ll}
b_{11}(t, x, \xi) & b_{12}(t, x, \xi) \\
b_{21}(t, x, \xi) & b_{22}(t, x, \xi)
\end{array}\right]=\sum_{j=1}^{N}\left[\begin{array}{cc}
b_{11 j}(t, x) & b_{12 j}(t, x) \\
b_{21 j}(t, x) & b_{22 j}(t, x)
\end{array}\right] \xi_{j}, \\
b_{m n j}(t, x) \in C^{1}\left([0, T] ; \mathscr{B}^{\infty}\right), \quad m, n=1,2, \quad j=1, \cdots, N .
\end{gathered}
$$

$\mathscr{B}^{\infty}=\mathscr{B}^{\infty}\left(\mathbf{R}^{N}\right)$ denotes the set of $C^{\infty}$-functions on $\mathbf{R}^{N}$ whose derivatives of any 
order are all bounded. It is convenient to use the notations

$$
b^{\text {diag }}(t, x, \xi)=\left[\begin{array}{cc}
b_{11}(t, x, \xi) & 0 \\
0 & b_{22}(t, x, \xi)
\end{array}\right], \quad b^{\text {off }}(t, x, \xi)=\left[\begin{array}{cc}
0 & b_{12}(t, x, \xi) \\
b_{21}(t, x, \xi) & 0
\end{array}\right]
$$

In applications to $(1.1)-(1.2)$, we see $(u, \bar{u})$ as $\left(v_{1}, v_{2}\right)$.

We here prepare pseudo-differential operators. Let $m \in \mathbb{R}$ and let $S^{m}$ be a class of symbols defined by

$$
S^{m} \equiv\left\{\left.p(x, \xi) \in C^{\infty}\left(\mathbb{R}^{N} \times \mathbf{R}^{N}\right)|| p\right|_{l} ^{(m)}<+\infty, \quad l=0,1,2, \cdots\right\}
$$

where

$$
\begin{gathered}
|p|_{l}^{(m)}=\sup _{\substack{|\alpha+\beta| \leqslant l \\
x, \xi \in \mathbf{R}^{N}}}\left|p_{(\beta)}^{(\alpha)}(x, \xi)\langle\xi\rangle-m+\right| \alpha||, \\
p_{(\beta)}^{(\alpha)}(x, \xi)=\partial_{\xi}^{\alpha} D_{x}^{\beta} p(x, \xi), \quad \alpha, \beta \in\left(\mathbf{Z}_{+}\right)^{N}, \quad D_{x}=-i \partial_{x}, \quad j=1, \cdots, N .
\end{gathered}
$$

$S^{m}$ is a Fréchet space with respect to the family of seminorms $|\cdot|_{l}^{(m)}, l=0,1,2$, $\cdots$. If $p(x, \xi) \in S^{m}$ is given, then $p(x, \xi)$ defines the operator $P$ by

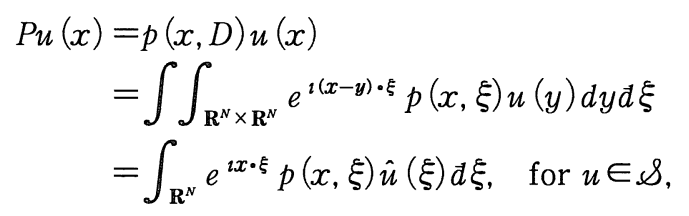

where $d \xi=(2 \pi)^{-N} d \xi$, and $\hat{u}(\xi)$ denotes the Fourier transform of $u(x)$. Conversely, if the operator $P$ is given, then its symbol $\sigma(P)(x, \xi)$ is calculated by

$$
\sigma(P)(x, \xi)=e^{-\imath x \cdot \xi} P\left(e^{i x \cdot \xi}\right) .
$$

The properties of pseudo-differential operators are the following.

\section{Lemma 2.1.}

(1) Let $p(x, \xi) \in S^{m}, m \in \mathbb{R}$. Then $P=p(x, D) \in \mathscr{L}\left(H^{s+m}, H^{s}\right)$ for any $s \in \mathbf{R}$ and there exist $l_{m}=l(m, N) \in \mathbb{N}$ and $C=C(s, N)>0$ such that

$$
\|P u\|_{s} \leqslant\left. C|p|\right|_{l m} ^{(m)}\|u\|_{s+m}, \quad \text { for } u \in H^{s+m}
$$

where $\mathscr{L}(X, Y)$ denotes the set of all bounded linear operators from the normed vector 
space $X$ to the normed vector space $Y$, and $\|\cdot\|_{s}$ is $H^{s}$-norm.

(2) Let $p(x, \xi) \in S^{m}$ and $p^{\prime}(x, \xi) \in S^{m^{\prime}}, m, m^{\prime} \in \mathbf{R}$, and let $\alpha, \alpha^{\prime}, \beta, \beta^{\prime} \in\left(\mathbf{Z}_{+}\right)^{N}$. We define

$$
r_{\theta}(x, \xi)=\mathrm{Os}-\iint_{\mathbf{R}^{N} \times \mathbf{R}^{N}} e^{-\imath y \cdot \eta} p_{(\beta)}^{(\alpha)}(x, \xi+\theta \eta) p^{\prime\left(\alpha^{\prime}\right)}(x+y, \xi) d y d \eta, \text { for } \theta \in[0,1],
$$

where "Os -" denotes oscillatory integrals (see H. Kumano-go [11]). Then, $\left\{r_{\theta}\right\}_{\theta \in[0,1]}$ is bounded in $S^{m+m^{\prime}-\left|\alpha+\alpha^{\prime}\right|}$ and for any $l \in \mathbf{N}$, there exist $l^{\prime} \in \mathbf{N}$ and $C_{1}>0$, which are independent of $\theta \in[0,1]$, such that

$$
\left|\gamma_{\theta}\right|_{l}^{\left(m+m^{\prime}-\left|\alpha+\alpha^{\prime}\right|\right)} \leqslant C_{l}|p|_{l^{\prime}}^{(m)}\left|p^{\prime}\right|_{l^{\prime}}^{\left(m^{\prime}\right)}
$$

(3) $\operatorname{Let} p(x, \xi) \in S^{m}$ and $p^{\prime}(x, \xi) \in S^{m^{\prime}}, m, m^{\prime} \in \mathbf{R}$. Then $\sigma\left(P P^{\prime}\right)(x, \xi) \in S^{m+m^{\prime}}$ and

$$
\sigma\left(P P^{\prime}\right)(x, \xi)=\mathrm{Os}-\iint_{\mathbb{R}^{N} \times \mathbf{R}^{N}} e^{-\imath y \cdot \eta} p(x, \xi+\eta) p^{\prime}(x+y, \xi) d y d \eta
$$

(4) (Sharp Gårding inequality) Let $p(x, \xi) \in S^{1}$ with $\operatorname{Re} p(x, \xi) \geqslant 0$, for $|\xi| \geqslant R \geqslant 0$. Then there exist $l \in \mathbf{N}$ and $C=C(R, N)>0$ such that

$$
\operatorname{Re}(P u, u) \geqslant-C|p|_{l}^{(1)}\|u\|^{2}, \text { for } u \in \&
$$

where $(\cdot, \cdot)$ and $\|\cdot\|$ denote $L^{2}$-inner product and $L^{2}$-norm respectively.

Concerning the details on pseudo-differential operators, see H. Kumanogo's textbook [11].

A simple sufficient condition of $L^{2}$-wellposedness for (2.1)-(2.2) is

Theorem 2.2. Assume that there exists a nonnegative and nonincreasing function $\gamma(s) \in \mathscr{B}^{\infty}[0, \infty) \cap L^{1}(0, \infty)$ with $d^{2 n+1} \gamma / d s^{2 n+1}(0)=0, n \in \mathbf{N}$ and there exists a nonnegative function $\phi(t) \in C^{1}[0, T]$ such that

$$
\left|\left(\operatorname{Im} b_{n n j}(t, x)\right)_{j=1, \cdots, N}\right| \leqslant \phi(t) \gamma(|x|), \quad n=1,2 \text {. }
$$

Then (2.1) - (2.2) is $L^{2}$-wellposed. Namely, for any $v_{0}(x) \in\left(L^{2}\right)^{2}$ and for any $f(t, x) \in\left(L_{\text {loc }}^{1}\left(0, T ; L^{2}\right)\right)^{2},(2.1)-(2.2)$ possesses a unique solution $v \in(C([0, T]$; $\left.\left.L^{2}\right)\right)^{2}$ which satisfies the following energy inequality 


$$
\|v(t)\| \leqslant C_{T}\left(\left\|v_{0}\right\|+\int_{0}^{t}\|f(\tau)\| d \tau\right), \quad \text { for } t \in[0, T]
$$

with some constant $C_{T}>0 .\|\cdot\|$ denotes $\left(L^{2}\right)^{2}{ }^{2}$ norm.

Our strategy divides into two steps. At the first step we diagonalize the operator $H(t)$ modulo bounded operators. Roughly speaking, its symbol

$$
h(t, x, \xi)=\left[\begin{array}{cc}
|\xi|^{2}+b_{11}(t, x, \xi) & b_{12}(t, x, \xi) \\
b_{21}(t, x, \xi) & -|\xi|^{2}+b_{22}(t, x, \xi)
\end{array}\right]
$$

has two distinct eigen-values provided that $|\xi|$ is sufficiently large. Thus we can easily diagonalize $h(t, x, \xi)$ and therefore this system becomes essentially same as two single equations. At the second step we apply S. Doi's method [3] to this diagonalized system.

Diagonalization. We define the following pseude-differential operators

$$
\begin{aligned}
& \tilde{\lambda}(t, x, \xi)=\left[\begin{array}{cc}
0 & \frac{1}{2} b_{12}(t, x, \xi)\langle\xi\rangle \\
-\frac{1}{2} b_{21}(t, x, \xi)\langle\xi\rangle^{-2} & 0
\end{array}\right] \in\left(C\left([0, T] ; S^{-1}\right)\right)^{2 \times 2}, \\
& \lambda(t, x, \xi)=I+\tilde{\lambda}(t, x, \xi), \quad \lambda^{\prime}(t, x, \xi)=I-\tilde{\lambda}(t, x, \xi) \in\left(C\left([0, T] ; S^{0}\right)\right)^{2 \times 2},
\end{aligned}
$$

where $I$ is the $2 \times 2$ identity matrix. Using $\lambda(t, x, \xi)$ and $\lambda^{\prime}(t, x, \xi)$, we can diagonalize $H(t)$ modulo bounded operators.

Lemma 2.3. Under the above assumptions, we have

$$
\sigma\left(\Lambda(t) H(t) \Lambda^{\prime}(t)\right)(x, \xi)-a(\xi)-b^{\mathrm{diag}}(t, x, \xi)=r_{1}(t, x, \xi) \in\left(C\left([0, T] ; S^{0}\right)\right)^{2 \times 2}
$$

where $\Lambda(t)=\lambda(t, x, D)$ and $\Lambda^{\prime}(t)=\lambda^{\prime}(t, x, D)$.

Proof. Direct calculations imply

$$
\begin{aligned}
\sigma\left(\Lambda(t) H(t) \Lambda^{\prime}(t)\right)(x, \xi)= & \sigma((I+\tilde{\Lambda}(t))(A+B(t))(I-\tilde{\Lambda}(t)))(x, \xi) \\
= & \sigma(A+\tilde{\Lambda}(t) A-A \tilde{\Lambda}(t)-\tilde{\Lambda}(t) A \tilde{\Lambda}(t))(x, \xi) \\
& +\sigma(B(t)+\tilde{\Lambda}(t) B(t)-B(t) \tilde{\Lambda}(t) \\
& -\tilde{\Lambda}(t) B(t) \tilde{\Lambda}(t))(x, \xi)
\end{aligned}
$$




$$
\begin{aligned}
= & a(\xi)+\sigma(\tilde{\Lambda}(t) A-A \tilde{\Lambda}(t))(x, \xi)+b(t, x, \xi) \\
& +r_{2}(t, x, \xi), \\
r_{2}(t, x, \xi)=- & \sigma(\tilde{\Lambda}(t) A \tilde{\Lambda}(t))(x, \xi)+\sigma(\tilde{\Lambda}(t) B(t)-B(t) \tilde{\Lambda}(t) \\
- & \tilde{\Lambda}(t) B(t) \tilde{\Lambda}(t))(x, \xi) .
\end{aligned}
$$

We have

$$
\begin{aligned}
& \begin{aligned}
\sigma(\tilde{\Lambda}(t) A-A \tilde{\Lambda}(t))(x, \xi) & =\tilde{\lambda}(t, x, \xi) a(\xi)-a(\xi) \tilde{\lambda}(t, x, \xi)+r_{3}(t, x, \xi) \\
& =-b^{\text {off }}(t, x, \xi)|\xi|^{2}\langle\xi\rangle^{-2}+r_{3}(t, x, \xi) \\
& =-b^{\text {off }}(t, x, \xi)+r_{4}(t, x, \xi)+r_{3}(t, x, \xi)
\end{aligned} \\
& r_{3}(t, x, \xi)=-\sum_{j=1}^{N} \int_{0}^{1}\left(\text { Os }-\iint e^{-\imath y \cdot \eta} a^{(j)}(\xi+\theta \eta) \tilde{\lambda}_{(j)}(t, x+y, \xi) d y d \eta\right) d \theta \\
& r_{4}(t, x, \xi)=b^{\text {off }}(t, x, \xi)\langle\xi\rangle^{-2}
\end{aligned}
$$

Substituting (2.6) into (2.4) and putting $r_{1}=r_{2}+r_{3}+r_{4}$, we obtain

$$
\begin{aligned}
\sigma\left(\Lambda(t) H(t) \Lambda^{\prime}(t)\right)(x, \xi) & =a(\xi)+b(t, x, \xi)-b^{\text {off }}(t, x, \xi)+r_{1}(t, x, \xi) \\
& =a(\xi)+b^{\text {diag }}(t, x, \xi)+r_{1}(t, x, \xi)
\end{aligned}
$$

It is easy to check $r_{1}(t, x, \xi) \in\left(C\left([0, T] ; S^{0}\right)\right)^{2 \times 2}$.

Now we will diagonalize the system (2.1) in some sense. Note that

$$
\Lambda^{\prime}(t) \Lambda(t)=(I-\tilde{\Lambda}(t))(I+\tilde{\Lambda}(t))=I-\tilde{\Lambda}^{2}(t) .
$$

Then

$$
\begin{aligned}
\Lambda(t) H(t) & =\Lambda(t) H(t)\left(\Lambda^{\prime}(t) \Lambda(t)+\tilde{\Lambda}^{2}(t)\right) \\
& =\Lambda(t) H(t) \Lambda^{\prime}(t) \Lambda(t)+\Lambda(t) H(t) \tilde{\Lambda}^{2}(t)
\end{aligned}
$$

Using Lemma 2.3, we have

$$
\begin{aligned}
\Lambda(t) H(t) & =\left(A+B^{\mathrm{diag}}(t)+R_{1}(t)\right) \Lambda(t)+\Lambda(t) H(t) \tilde{\Lambda}^{2}(t) \\
& =\left(A+B^{\mathrm{diag}}(t)\right) \Lambda(t)-i \tilde{C}(t),
\end{aligned}
$$

where we put $\tilde{C}(t)=i\left(R_{1}(t) \Lambda(t)+\Lambda(t) H(t) \tilde{\Lambda}^{2}(t)\right)$. By using (2.5), (2.7) and $(2.9)$, it is easy to see $\tilde{c}(t, x, \xi) \in\left(C\left([0, T] ; S^{0}\right)\right)^{2 \times 2}$ and there exist $l \in \mathbf{N}$ and $C>0$ such that 


$$
|\tilde{c}(t)|_{l_{0}}^{(0)} \leqslant C\left(\max _{m, n, j} \mid b_{m n j}(t) \|_{\mathscr{B}^{b}}+\left(\max _{m, n, j}\left\|b_{m n j}(t)\right\|_{\mathscr{B g}^{\prime}}\right)^{4}\right) .
$$

Here

$$
\mathscr{B}^{l}=\mathscr{B}^{l}\left(\mathbf{R}^{N}\right) \equiv\left\{u \in C^{l}\left(\mathbf{R}^{N}\right)\left|\|u\|_{\mathscr{B}}=\sum_{|\alpha| \leqslant l} \sup _{\mathbf{R}^{N}}\right| u(x) \mid<+\infty\right\} .
$$

Operating $\Lambda(t)$ on (2.1), we have

$$
\partial_{t}(\Lambda(t) v)+i \Lambda(t) H(t) v-\Lambda_{t}(t) v=\Lambda(t) f
$$

where

$$
\begin{gathered}
\sigma\left(\Lambda_{t}(t)\right)(x, \xi)=\partial_{t} \lambda(t, x, \xi)=\partial_{t} \tilde{\lambda}(t, x, \xi) \in\left(C\left([0, T] ; S^{-1}\right)\right)^{2 \times 2}, \\
\left|\partial_{t} \tilde{\lambda}(t)\right|_{l_{-1}}^{(-1)} \leqslant C \max _{m, n, j}\left\|\partial_{t} b_{m n j}(t)\right\|_{\mathscr{B}^{t}}
\end{gathered}
$$

Substituting (2.9) into (2.11), we obtain

$$
\partial_{t}(\Lambda(t) v)+i\left(A+B^{\mathrm{diag}}(t)\right)(\Lambda(t) v)+\left(\tilde{C}(t)-\Lambda_{t}(t)\right) v=\Lambda(t) f
$$

Our diagonalization is completed.

Proof of Theorem 2.2. The system (2.13) is a couple of single equations essentially. Thus we can apply the theory of linear Schrödinger type equations. The following arguments in this subsection are basically due to S. Doi [3].

First we define pseudo-differential operators which eliminate bad first order terms in some sense.

$$
\begin{aligned}
& \left.p(t, x, \xi)=\phi(t) \sum_{j=1}^{N}\left(\int_{0}^{x} \gamma(s) d s\right) \xi_{j}\langle\xi\rangle\right\rangle^{-1} \in C\left([0, T] ; S^{0}\right), \\
& k(t, x, \xi)=\left[\begin{array}{cc}
e^{-p(t, x, \xi)} & 0 \\
0 & e^{p(t, x, \xi)}
\end{array}\right] \in\left(C\left([0, T] ; S^{0}\right)\right)^{2 \times 2}, \\
& k^{\prime}(t, x, \xi)=\left[\begin{array}{cc}
e^{p(t, x, \xi)} & 0 \\
0 & e^{-p(t, x, \xi)}
\end{array}\right] \in\left(C\left([0, T] ; S^{0}\right)\right)^{2 \times 2},
\end{aligned}
$$

where $\gamma(s),(s \in \mathbf{R})$, is extended to $\mathbf{R}$ as an even function. Since we assume $d^{2 n+1} \gamma / d s^{2 n+1}(0)=0, n \in \mathbb{N}$, the extension $\gamma(s)$ belongs to $\mathscr{B}^{\infty}(\mathbf{R})$. Properties of the transformation $K(t) \Lambda(t)=k(t, x, D) \lambda(t, x, D)$ are the following. 
Lemma 2.4. There exist $l \in \mathbf{N}$ and $C_{1}>0$ such that

$$
\|v\| \leqslant C_{K}^{2}(t)\left(1+C_{B}^{2}(t)\right)\left(\|K(t) \Lambda(t) v\|+\|v\|_{-1}\right) \leqslant C_{K}^{3}(t)\left(1+C_{B}^{3}(t)\right)\|v\|
$$

for any $v \in\left(L^{2}\right)^{2}$, where

$$
C_{K}(t)=C_{1}|k(t)|_{l}^{(0)}, \quad C_{B}(t)=C_{1}\left(\max _{m, n, j}\left\|b_{m n j}(t)\right\|_{\mathscr{B}^{i}}+\max _{m, n, j}\left\|\partial_{t} b_{m n j}(t)\right\|_{\mathscr{B}^{\prime}}\right) .
$$

Proof. Direct calculations give

$$
\begin{aligned}
I & =\Lambda^{\prime}(t) K^{\prime}(t) K(t) \Lambda(t)+\tilde{\Lambda}(t)^{2}+\Lambda^{\prime}(t) R_{0}(t) \Lambda(t), \\
\sigma\left(R_{0}(t)\right)(x, \xi) & =\sigma\left(I-K^{\prime}(t) K(t)\right)(x, \xi) \\
& =-\sum_{j=1}^{N} \int_{0}^{1} \iint_{\mathbf{R}^{N} \times \mathbf{R}^{N}} e^{-\imath y \cdot \eta} k^{(j)}(t, x, \xi+\theta \eta) k_{(j)}(t, x+y, \xi) d y d \eta d \theta .
\end{aligned}
$$

Then it is easy to verify Lemma 2.4.

Theorem 2.2 follows from the next energy inequality with standard arguments (see L. Hörmander [7, §23.1] for instance).

Lemma 2.5. Let $\left.v \in\left(C[0, T] ; H^{2}\right) \cap C^{1}\left([0, T] ; L^{2}\right)\right)^{2}$, then there exists a constant $C_{T}>0$ such that

$$
\|v(t)\| \leqslant C_{T}\left(\|v(0)\|+\int_{0}^{t}\left\|\left(\partial_{t}+i H(\tau)\right) v(\tau)\right\| d \tau\right), \quad \text { for } \quad t \in[0, T]
$$

Proof. Put $f=\left(\partial_{t}+i H(t)\right) v$, then $f(t, x) \in\left(C\left([0, T] ; L^{2}\right)^{2}\right.$. Let $N(v)=$ $\|K(t) \Lambda(t) v\|+\|v\|_{-1}$. Lemma 2.4 indicates that $N(v)$ is equivalent to $\left(L^{2}\right)^{2}$-norm for each $t \in[0, T]$. Hence we have only to obtain the energy inequality with respect to $N(v)$. We denote $\left(L^{2}\right)^{2}$-inner product by $(\cdot, \cdot)$. We have

$$
\begin{aligned}
\frac{d}{d t}\|K(t) \Lambda(t) v\|^{2} & =2 \operatorname{Re}\left(\partial_{t}(K(t) \Lambda(t) v), K(t) \Lambda(t) v\right) \\
& =2 \operatorname{Re}\left(K_{t}(t) \Lambda(t) v+K(t) \partial_{t}(\Lambda(t) v), K(t) \Lambda(t) v\right)
\end{aligned}
$$

(2.13) gives

$$
\frac{d}{d t}\|K(t) \Lambda(t) v\|^{2}=-2 \operatorname{Re}\left(i K(t)\left(A+B^{\mathrm{diag}}(t)\right) \Lambda(t) v, K(t) \Lambda(t) v\right)
$$




$$
\begin{aligned}
& +2 \operatorname{Re}\left(\left(K_{t}(t) \Lambda(t)+K(t)\left(\tilde{C}(t)-\Lambda_{t}(t)\right)\right) v, K(t) \Lambda(t) v\right) \\
& +2 \operatorname{Re}(K(t) \Lambda(t) f, K(t) \Lambda(t) v),
\end{aligned}
$$

where

$$
\begin{aligned}
& \sigma\left(K_{t}(t)\right)(x, \xi)=\partial_{t} k(t, x, \xi)=\phi^{\prime}(t) \sum_{j=1}^{N}\left(\int_{0}^{x_{j}} \gamma(s) d s\right) \xi_{j}\langle\xi\rangle^{-1}\left[\begin{array}{cc}
-e^{-p(t, x, \xi)} & 0 \\
0 & e^{p(t, x, \xi)}
\end{array}\right] \\
& \left|\sigma\left(K_{t}(t)\right)\right|_{l_{0}}^{(0)} \leqslant\left|\phi^{\prime}(t)\right| C_{r} C_{K}(t), \quad C_{r}=C_{1}\left(\|\gamma\|_{\mathscr{B}^{\prime}}+\|\gamma\|_{L^{1}}\right) .
\end{aligned}
$$

Using (2.10), (2.12) and (2.14), we get

$$
\begin{aligned}
\frac{d}{d t}\|K(t) \Lambda(t) v\|^{2} & \leqslant-2 \operatorname{Re}\left(i K(t)\left(A+B^{\mathrm{diag}}(t)\right) \Lambda(t) v, K(t) \Lambda(t) v\right) \\
& +\left(C_{B}(t)+\left|\phi^{\prime}(t)\right|\right) C_{2}(t)\|v\|\|K(t) \Lambda(t) v\| \\
& +2\|K(t) \Lambda(t) f\|\|K(t) \Lambda(t) v\|, \\
C_{2}(t)= & \left(1+C_{K}(t)\right)\left(1+C_{B}^{3}(t)\right)\left(1+C_{\tau}^{2}\right)(1+\phi(t)) .
\end{aligned}
$$

We denote Poisson's bracket by $\left\{\cdot,^{\circ}\right\}$. Noting that $k(x, \xi)$ and $a(\xi)+b^{\text {diag }}(x, \xi)$ are diagonal matrices, we have

$$
\begin{aligned}
& \sigma\left(i K(t)\left(A+B^{\text {diag }}(t)\right)\right)(x, \xi) \\
& =\sigma\left(i\left(A+B^{\mathrm{diag}}(t)\right) K(t)\right)(x, \xi) \\
& +\sigma\left(\left[\begin{array}{cc}
i\left[e^{-p(t, x, D)},|D|^{2}+b_{11}(t, x, D)\right] & 0 \\
0 & i\left[e^{p(t, x, D)},-|D|^{2}+b_{22}(t, x, D)\right]
\end{array}\right]\right)(x, \xi) \\
& =\sigma\left(i\left(A+B^{\mathrm{diag}}(t)\right) K(t)\right)(x, \xi) \\
& +\sigma\left(\left[\begin{array}{cc}
i\left[e^{-p(t, x, D)},|D|^{2}\right] & 0 \\
0 & i\left[e^{p(t, x, D)},-|D|^{2}\right]
\end{array}\right]\right)(x, \xi) r_{7}(t, x, \xi) \\
& =\sigma\left(i\left(A+B^{\text {diag }}(t)\right) K(t)\right)(x, \xi)+\left\{|\xi|^{2}, p\right\}(t, x, \xi) k(t, x, \xi) \\
& +r_{8}(t, x, \xi)+r_{7}(t, x, \xi) \\
& =\sigma\left(i\left(A+B^{\mathrm{diag}}(t)\right) K(t)\right)(x, \xi)+2 \phi(t) \sum_{j=1}^{N} \gamma\left(x_{j}\right) \xi_{j}^{2}\langle\xi\rangle^{-1} k(t, x, \xi) \\
& +r_{8}(t, x, \xi)+r_{7}(t, x, \xi) \\
& =\sigma\left(\left(i\left(A+B^{\mathrm{diag}}(t)\right)+2 \phi(t) \sum_{j=1}^{N} \gamma\left(x_{j}\right) D_{x}^{2}\langle D\rangle^{-1}\right) K(t)\right)(x, \xi) \\
& +r_{9}(t, x, \xi)+r_{8}(t, x, \xi)+r_{7}(t, x, \xi) \text {, }
\end{aligned}
$$

where 


$$
\begin{aligned}
& r_{7}(t, x, \xi)=i \sum_{j=1}^{N} \int_{0}^{1}\left(\text { Os }-\iint e^{-i y \cdot \eta} \quad\left(k^{(j)}(t, x, \xi+\theta \eta) b_{(j)}^{\mathrm{diag}}(t, x+y, \xi)\right.\right. \\
& \left.\left.-b^{\mathrm{diag}(\omega)}(t, x, \xi+\theta \eta) k_{(j)}(t, x+y, \xi)\right) d y d \eta\right) d \theta \\
& r_{8}(t, x, \xi)=-i \sum_{j=1}^{N} \tilde{J} k_{(j j)}(t, x, \xi), \quad \tilde{J}=\left[\begin{array}{cc}
1 & 0 \\
0 & -1
\end{array}\right] \\
& r_{9}(t, x, \xi)=-2 \phi(t) \sum_{j, n=1}^{N} \int_{0}^{1}\left\{\mathrm{Os}-\iint e^{-i y \cdot \eta} \gamma\left(x_{j}\right)\left(2\left(\xi_{j}+\theta \eta_{j}\right)\langle\xi+\theta \eta\rangle^{-1} \delta_{j n}\right.\right. \\
& \left.\left.\quad-\left(\xi_{j}+\theta \eta_{j}\right)^{2}\left(\xi_{n}+\theta \eta_{n}\right)\langle\xi+\theta \eta\rangle^{-3}\right) k_{(n)}(t, x+y, \xi) d y d \eta\right\} d \theta
\end{aligned}
$$

and they are estimated by

$$
r_{7}, r_{8}, r_{9} \in\left(C\left([0, T] ; S^{0}\right)\right)^{2 \times 2}, \quad\left|r_{7}(t)\right|_{l_{0}}^{(0)},\left|r_{8}(t)\right|_{l_{0}}^{(0)},\left|r_{9}(t)\right|_{l_{0}}^{(0)} \leqslant \phi(t) C_{2}(t)
$$

Substitute (2.17) into (2.15), then

$$
\begin{aligned}
& \frac{d}{d t}\|K(t) \Lambda(t) v\|^{2} \\
& \leqslant-2 \operatorname{Re}\left(\left(i\left(A+B^{\mathrm{diag}}(t)\right)+2 \phi(t) \sum_{j=1}^{N} \gamma\left(x_{j}\right) D_{x}^{2}\langle D\rangle^{-1}\right) K(t) \Lambda(t) v, K(t) \Lambda(t) v\right) \\
& +\left(\phi(t)+\left|\phi^{\prime}(t)\right|+C_{B}(t)\right)\|v\|\|K(t) \Lambda(t) v\|+2\|K(t) \Lambda(t) f\|\|K(t) \Lambda(t) v\| .
\end{aligned}
$$

Lemma 2.4 implies

$$
\begin{aligned}
& \frac{d}{d t}\|K(t) \Lambda(t) v\|^{2} \\
& \leqslant-2 \operatorname{Re}\left(\left(i\left(A+B^{\mathrm{diag}}(t)\right)+2 \phi(t) \sum_{j=1}^{N} \gamma\left(x_{j}\right) D_{x}^{2}\langle D\rangle^{-1}\right) K(t) \Lambda(t) v, K(t) \Lambda(t) v\right) \\
& +\left(\phi(t)+\left|\phi^{\prime}(t)\right|+C_{B}(t)\right) C_{2}(t) N(v)\|K(t) \Lambda(t) v\|+2\|K(t) \Lambda(t) f\|\|K(t) \Lambda(t) v\| \\
& =-2 \operatorname{Re} \sum_{n=1,2}\left(\sum_{j=1}^{N}\left(2 \phi(t) \gamma\left(x_{j}\right) D_{x}^{2}\langle D\rangle^{-1}+i b_{n n j}(t, x) D_{x}\right)(K(t) \Lambda(t) v)_{n},(K(t) \Lambda(t) v)_{n}\right) \\
& +\left(\phi(t)+\left|\phi^{\prime}(t)\right|+C_{B}(t)\right) C_{2}^{3}(t) N(v)\|K(t) \Lambda(t) v\|+2\|K(t) \Lambda(t) f\|\|K(t) \Lambda(t) v\| .
\end{aligned}
$$

Since (2.3) yields

$$
\operatorname{Re} \sum_{j=1}^{N}\left(2 \phi(t) \gamma\left(x_{j}\right) \xi_{j}^{2}\langle\xi\rangle^{-1}+i b_{n n j}(t, x) \xi_{j}\right) \geqslant 0, \quad \text { for }|\xi| \geqslant 1, n=1,2
$$

sharp Gårding inequality implies 


$$
\begin{aligned}
\frac{d}{d t}\|K(t) \Lambda(t) v\|^{2} & \leqslant\left(\phi(t)+\left|\phi^{\prime}(t)\right|+C_{B}(t)\right) C_{2}^{3}(t) N(v)\|K(t) \Lambda(t) v\| \\
& +2\|K(t) \Lambda(t) f\|\|K(t) \Lambda(t) v\|,
\end{aligned}
$$

namely

$$
\frac{d}{d t}\|K(t) \Lambda(t) v\| \leqslant\left(\phi(t)+\left|\phi^{\prime}(t)\right|+C_{B}(t)\right) C_{2}^{3}(t) N(v)+\|K(t) \Lambda(t) f\| .
$$

(2.18) will be very useful later. On the other hand, we can get

$$
\frac{d}{d t}\|v\|_{-1} \leqslant C_{B}(t)\|v\|+\|f\|_{-1} \leqslant C_{B}(t) C_{2}^{2}(t) N(v)+\|f\|_{-1}
$$

Combining (2.18) and (2.19), we obtain

$$
\frac{d}{d t} N(v) \leqslant\left(\phi(t)+\left|\phi^{\prime}(t)\right|+C_{B}(t)\right) C_{2}^{3}(t) N(v)+N(f) .
$$

\section{§3. Preliminaries}

In this section we present the local existence theorem, chain-rule with respect to $J$ and some embedding lemma.

Concerning the local existence, we have

Proposition 3.1. Assume (1.3) and (1.4) with $p_{w}=3$. Let $m$ be a sufficiently large integer. Then for any $u_{0} \in \cap_{j=0}^{1} H^{m-j, j}$, there exists a time $T$ $=T\left(\left\|u_{0}\right\|_{\cap_{j=0}^{1} H^{m-s, s}}\right)>0$ such that $(1.1)-(1.2)$ possesses a unique solution $u \in$ $\bigcap_{j=0}^{1} C\left([0, T) ; H^{m-j, j}\right)$.

Proof. Following [2], we give the outline of proof. First we consider

$$
\begin{gathered}
\partial_{t} u^{\varepsilon}-(\varepsilon+i) \Delta u^{\varepsilon}=F\left(u^{\varepsilon}, \nabla u^{\varepsilon}\right), \text { in }(0, \infty) \times \mathbb{R}^{N}, \\
u^{\varepsilon}(0, x)=u_{0}(x), \text { in } \mathbb{R}^{N},
\end{gathered}
$$

with $\varepsilon \in(0,1]$. We can easily solve (3.1) - (3.2) because the parabolic regularization overcomes the loss of derivatives. The existence time depends on $\varepsilon \in$ $(0,1]$. But similarly to $\S 2$ (see also $\S 4$ ), we can show that there exists a time $T>0$, which is independent of $\varepsilon \in(0,1]$, such that $\left\{u^{\varepsilon}\right\}_{\varepsilon \in(0,1]}$ is bounded in $\bigcap_{j=0}^{1} L^{\infty}\left(0, T ; H^{m-j, j}\right)$. Then using the standard compactness arguments, we can get a solution $u \in \cap{ }_{j=0}^{1} L^{\infty}\left(0, T ; H^{m-j, j}\right)$ provided $\varepsilon \downarrow 0$. The uniqueness and the 
time continuity can be also proved by the same energy method.

We shall prove Theorem 1.1 by a priori estimates besed on the energy inequality obtained in $\S 2$. Then we need twice more regularity of the solution for the validity of the energy inequality (see Lemma 2.5). Let us consider the following situation. Under the same assumption of Proposition 3.1, let $u_{0} \in$ $\bigcap_{j=0}^{1} H^{m+1-j, j}$ and let $u$ be a solution to (1.1)-(1.2). Proposition 3.1 gives

$$
T_{0} \equiv T\left(\left\|u_{0}\right\|_{\cap_{j=0}^{1} H^{m-, j}}\right) \geqslant T_{1} \equiv T\left(\left\|u_{0}\right\|_{\Gamma_{j=0}^{1} H^{m+1-J, J}}\right)>0,
$$

such that

$$
u \in \bigcap_{j=0}^{1} C\left(\left[0, T_{0}\right) ; H^{m-j, j}\right) \text { and } u \in \bigcap_{j=0}^{1} C\left(\left[0, T_{1}\right) ; H^{m+1-j, j}\right) .
$$

Moreover we obtain

Lemma 3.2. Under the above assumption, $T_{0}=T_{1}$ holds.

Proof. Assume $T_{0}>T_{1}$, then Proposition 3.1 implies

$$
\lim _{t \uparrow T 1}\|u(t)\|_{\cap_{j=0}^{1} H^{m+1-1,}}=+\infty
$$

We will derive a contradiction.

Put $R=\max _{t \in\left[0, T_{2}\right]}\|u(t)\|_{\Gamma_{j=0}^{1} H^{m-\jmath} \text {. Let }} \varphi_{k}(x)$ and $\alpha \in\left(\mathbf{Z}_{+}\right)^{N}$ satisfy

$$
\varphi_{k}(x)=\left\{\begin{array}{ll}
1, & (k=0), \\
x_{k}, & (k=1, \cdots, N),
\end{array} \quad|\alpha|= \begin{cases}m, & (k=0), \\
m-1, & (k=1, \cdots, N)\end{cases}\right.
$$

Let $l$ be the same integer as in $\S 2$. Operating $\varphi_{k}(x) \partial_{x}^{\alpha}$ on $(1.1)-(1.2)$ and using Leibniz' formula, we have

$$
\begin{gathered}
\partial_{t}\left(\varphi_{k}(x) \partial_{x}^{\alpha} u\right)-i \Delta\left(\varphi_{k}(x) \partial_{x}^{\alpha} u\right)-i \sum_{j=1}^{N} \frac{\partial F}{\partial q_{j}} D_{x}\left(\varphi_{k}(x) \partial_{x}^{\alpha} u\right)-i \sum_{j=1}^{N} \frac{\partial F}{\partial \bar{q}_{j}} D_{x}\left(\varphi_{k}(x) \partial_{x}^{\alpha} \bar{u}\right) \\
+\sum_{n=0}^{N} \sum_{\beta} a_{n \beta}^{k \alpha}(u) \varphi_{n}(x) \partial_{x}^{\beta} u+\sum_{n=0}^{N} \sum_{\beta} b_{n \beta}^{k \alpha}(u) \varphi_{n}(x) \partial_{x}^{\beta} \bar{u}=G^{k \alpha}(u)
\end{gathered}
$$

where $\sum_{n=0}^{N} \sum_{\beta}$ means summing up on $n, \beta$ satisfying (3.3). It is easy to see

$$
\sup _{t \in[0, T 11]} \max _{k, \alpha, n, \beta}\left(\left\|\alpha_{n \beta}^{k \alpha}(u)\right\|_{\mathscr{B}^{1}}+\left\|\partial_{t} a_{n \beta}^{k \alpha}(u)\right\|_{\mathscr{B}^{t}}+\left\|b_{n \beta}^{k \alpha}(u)\right\|_{\mathscr{B}^{t}}+\left\|\partial_{t} b_{n \beta}^{k \alpha}(u)\right\|_{\mathscr{B}^{t}}+\left\|G^{k \alpha}(u)\right\|_{H^{1}}\right) \leqslant C_{R^{\prime}}
$$


Now let us consider the following system

$$
\begin{gathered}
\partial_{t} w_{k \alpha}-i \Delta w_{k \alpha}-i \sum_{j=1}^{N} \frac{\partial F}{\partial q_{j}} D_{x}, w_{k \alpha}-i \sum_{j=1}^{N} \frac{\partial F}{\partial \bar{q}_{j}} D_{x}, \bar{w}_{k \alpha} \\
+\sum_{n=0}^{N} \sum_{\beta} a_{n \beta}^{k \alpha}(u) w_{n \beta}+\sum_{n=0}^{N} \sum_{\beta} b_{n \beta}^{k \alpha}(u) \bar{w}_{n \beta}=G^{k \alpha}(u), \text { in }\left[0, T_{1}\right] \times \mathbb{R}^{N}, \\
w_{k \alpha}=\varphi_{k}(x) \partial_{x}^{\alpha} u_{0}(x) \text { in } \mathbf{R}^{N} .
\end{gathered}
$$

We see $(3.4)-(3.5)$ as a system of $\left\{\left(w_{k \alpha}, \bar{w}_{k \alpha}\right)\right\}_{k, \alpha}$. Then similarly to the proof

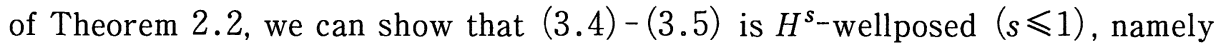
we get

$$
\left\|w_{k \alpha}(t)\right\|_{1} \leqslant C_{R}\left(\left\|\varphi_{k}(x) \partial_{x}^{\alpha} u_{0}\right\|_{1}+T_{1}\right), t \in\left[0, T_{1}\right], w_{k \alpha}=\varphi_{k}(x) \partial_{x}^{\alpha} u \text { in } C\left(\left[0, T_{1}\right) ; H^{1}\right)
$$

Hence we obtain lim $\sup _{t \uparrow T_{1}}\left\|\varphi_{k}(x) \partial_{x}^{\alpha} u(t)\right\|_{1}<+\infty$.

Now we consider the chain-rule of $J$.

Lemma 3.3. Assume (1.3) and (1.7). Let $\alpha, \beta \in\left(\mathbf{Z}_{+}\right)^{N}$. Then we have

$$
\begin{aligned}
& \partial_{x}^{\alpha} J^{\beta} F(u, \nabla u)=\sum_{*}\left(\frac{\partial}{\partial u}\right)^{l_{0}}\left(\frac{\partial}{\partial \bar{u}}\right)^{\bar{l}_{0}}\left(\frac{\partial}{\partial q}\right)^{l}\left(\frac{\partial}{\partial \bar{q}}\right)^{\bar{l}} F(u, \nabla u) \\
& \times \prod_{\substack{\alpha_{0} \leqslant \alpha \\
\beta_{0} \leqslant \beta}}\left(\partial_{x}^{\alpha_{0}} J^{\beta_{0}} u\right)^{m\left(\alpha_{0}, \beta_{0}\right)} \\
& \times \prod_{j=1}^{N} \prod_{\substack{\alpha, \leqslant \alpha \\
\beta, \leqslant \beta}}\left(\partial_{x}^{\alpha_{j}} j^{\beta,} \partial_{x j} u\right)^{m(\alpha, \beta,)} \\
& \times \prod_{\substack{\alpha_{0} \leqslant \alpha \\
\bar{\beta}_{0} \leqslant \beta}}(-1)^{\left|\bar{\beta}_{0}\right| m\left(\bar{\alpha}_{0}, \bar{\beta}_{0}\right)}\left(\overline{\partial_{x}^{\bar{\alpha}_{0}} J^{\bar{\beta}_{0}} u}\right)^{m\left(\bar{\alpha}_{0}, \bar{\beta}_{0}\right)} \\
& \times \prod_{j=1}^{N} \prod_{\substack{\bar{\alpha}_{j} \leqslant \alpha \\
\bar{\beta}, \leqslant \beta}}(-1)^{|\bar{\beta}| \mid m(\bar{\alpha},, \bar{\beta},)}\left(\overline{\partial_{x}^{\bar{\alpha}}, J^{\bar{\beta}}, \partial_{x j} u}\right)^{m\left(\bar{\alpha}, \bar{\beta}_{j}\right)},
\end{aligned}
$$

for $u \in C^{\infty}\left(\mathbf{R}^{N}\right)$, where $l_{0}, \bar{l}_{0}, m\left(\alpha_{n}, \beta_{n}\right), m\left(\bar{\alpha}_{n}, \bar{\beta}_{n}\right) \in \mathbb{Z}_{+}, l, \bar{l}, \alpha_{n}, \beta_{n}, \bar{\alpha}_{n}, \bar{\beta}_{n} \in\left(\mathbb{Z}_{+}\right)^{N}$, $n=0,1, \cdots, N$ and $\sum_{*}$ means taking $\sum_{1 \leqslant l_{0}+\bar{l}_{0}+l+\bar{l} \leqslant|\alpha+\beta|}$ restricted to

$$
\begin{array}{ll}
\sum_{\substack{\alpha_{0} \leqslant \alpha \\
\beta_{0} \leqslant \beta}} m\left(\alpha_{0}, \beta_{0}\right)=l_{0}, \quad \sum_{j=1}^{N} \sum_{\substack{\alpha, \leqslant \alpha \\
\beta, \leqslant \beta}} m\left(\alpha_{j}, \beta_{j}\right)=|l|, \\
\sum_{\substack{\alpha_{0} \leqslant \alpha \\
\bar{\beta}_{0} \leqslant \beta}} m\left(\bar{\alpha}_{0}, \bar{\beta}_{0}\right)=\bar{l}_{0}, \quad \sum_{j=1}^{N} \sum_{\substack{\alpha_{j} \leqslant \alpha \\
\bar{\beta}, \leqslant \beta}} m\left(\bar{\alpha}_{j}, \bar{\beta}_{j}\right)=|\bar{l}|,
\end{array}
$$




$$
\begin{array}{r}
\sum_{\substack{\alpha_{0} \leqslant \alpha \\
\beta_{0} \leqslant \beta}} m\left(\alpha_{0}, \beta_{0}\right)\left[\begin{array}{c}
\alpha_{0} \\
\beta_{0}
\end{array}\right]+\sum_{j=1}^{N} \sum_{\substack{\alpha \leqslant \alpha \\
\beta, \leqslant \beta}} m\left(\alpha_{j}, \beta_{j}\right)\left[\begin{array}{c}
\alpha_{j} \\
\beta_{j}
\end{array}\right] \\
+\sum_{\substack{\bar{\alpha}_{0} \leqslant \alpha \\
\bar{\beta}_{0} \leqslant \beta}} m\left(\bar{\alpha}_{0}, \bar{\beta}_{0}\right)\left[\begin{array}{l}
\bar{\alpha}_{0} \\
\bar{\beta}_{0}
\end{array}\right]+\sum_{j=1}^{N} \sum_{\substack{\bar{\alpha}_{s} \leqslant \alpha \\
\bar{\beta}_{j} \leqslant \beta}} m\left(\bar{\alpha}_{j}, \bar{\beta}_{j}\right)\left[\begin{array}{c}
\bar{\alpha}_{j} \\
\bar{\beta}_{j}
\end{array}\right]=\left[\begin{array}{c}
\alpha \\
\beta
\end{array}\right] .
\end{array}
$$

Proof. We have only to prove (3.6) with $\alpha=0$ because the usual Leibniz' formula extends $(3.6)$ to the case of general $\alpha(\neq 0)$.

Noting the gauge invariance (1.7) and differentiation to composition, we get

$$
\begin{gathered}
e^{i \theta\left(l_{0}+|l|-\bar{l}_{0}-|\bar{l}|\right)}\left\{\left(\frac{\partial}{\partial u}\right)^{l_{0}}\left(\frac{\partial}{\partial \bar{u}}\right)^{\bar{l}_{0}}\left(\frac{\partial}{\partial q}\right)^{l}\left(\frac{\partial}{\partial \bar{q}}\right)^{\bar{l}} F\right\}\left(e^{i \theta} u, e^{i \theta} q\right) \\
=e^{i \theta}\left(\frac{\partial}{\partial u}\right)^{l_{0}}\left(\frac{\partial}{\partial \bar{u}}\right)^{\bar{l}_{0}}\left(\frac{\partial}{\partial q}\right)^{l}\left(\frac{\partial}{\partial q}\right)^{\bar{l}} F(u, q)
\end{gathered}
$$

for $(u, q) \in \mathbf{C} \times \mathbf{C}^{N}, \theta \in \mathbf{R}$.

Now we put $\theta=|x|^{2} / 4(1+t)$ for convenience. We have

$$
\begin{aligned}
J^{\beta} F(u, \nabla u) & =\left(e^{i \theta} 2 i(1+t) \partial_{x} e^{-\imath \theta}\right)^{\beta} F(u, \nabla u) \\
& =e^{i \theta}(2 i(1+t))^{|\beta|} \partial_{x}^{\beta}\left(e^{-i \theta} F(u, \nabla u)\right) \\
& \left.=e^{i \theta}(2 i(1+t))\right)^{|\beta|} \partial_{x}^{\beta} F\left(e^{-\imath \theta} u, e^{-\imath \theta} \nabla u\right) \quad(\text { by }(1.7)) .
\end{aligned}
$$

Leibniz' formula gives

$$
\begin{aligned}
J^{\beta} F(u, \nabla u) & =e^{i \theta}(2 i(1+t))^{|\beta|} \sum_{*}\left\{\left(\frac{\partial}{\partial u}\right)^{l_{0}}\left(\frac{\partial}{\partial \bar{u}}\right)^{\bar{l}_{0}}\left(\frac{\partial}{\partial q}\right)^{l}\left(\frac{\partial}{\partial \bar{q}}\right)^{\bar{l}} F\right\}\left(e^{-i \theta} u, e^{-i \theta} \nabla u\right) \\
& \times \prod_{\beta_{0} \leqslant \beta}\left(\partial_{x}^{\beta_{0}}\left(e^{-i \theta} u\right)\right)^{m\left(\beta_{0}\right)} \times \prod_{j=1}^{N} \prod_{\beta, \leqslant \beta}\left(\partial_{x}^{\beta,}\left(e^{-i \theta} \partial_{x}, u\right)\right)^{m\left(\beta_{s}\right)} \\
& \times \prod_{\bar{\beta}_{0} \leqslant \beta}\left(\overline{\partial_{x}^{\bar{\beta}_{0}}\left(e^{-i \theta} u\right)}\right)^{m\left(\bar{\beta}_{0}\right)} \times \prod_{j=1}^{N} \prod_{\bar{\beta}_{\beta} \leqslant \beta}\left(\overline{\partial_{x}^{\bar{\beta}},\left(e^{-i \theta} \partial_{x} u\right)}\right)^{m(\bar{\beta},)} .
\end{aligned}
$$

By using (3.9), then

$$
\begin{aligned}
J^{\beta} F(u, \nabla u)= & e^{i \theta\left(l_{0}+l-\bar{l}_{0}-|\bar{l}|\right)}(2 i(1+t))^{|\beta|} \sum_{*}\left(\frac{\partial}{\partial u}\right)^{l_{0}}\left(\frac{\partial}{\partial \bar{u}}\right)^{\bar{l}_{0}}\left(\frac{\partial}{\partial q}\right)^{l}\left(\frac{\partial}{\partial \bar{q}}\right)^{\bar{l}} F(u, q) \\
& \times \prod_{\beta_{0} \leqslant \beta}\left(\partial_{x}^{\beta_{0}}\left(e^{-\imath \theta} u\right)\right)^{m\left(\beta_{0}\right)} \times \prod_{j=1 \beta_{j} \leqslant \beta}^{N}\left(\partial_{x}^{\beta,}\left(e^{-\imath \theta} \partial_{x} u\right)\right)^{m(\beta,)} \\
& \times \prod_{\bar{\beta}_{0} \leqslant \beta}\left(\overline{\partial_{x}^{\bar{\beta}_{0}}\left(e^{-i \theta} u\right)}\right)^{m\left(\bar{\beta}_{0}\right)} \times \prod_{j=1 \bar{\beta}_{,} \leqslant \beta}^{N}\left(\overline{\partial_{x}^{\bar{\beta},}\left(e^{-\imath \theta} \partial_{x,} u\right)}\right)^{m(\bar{\beta},)} .
\end{aligned}
$$


Noting (3.7) and (3.8), we obtain

$$
\begin{aligned}
& J^{\beta} F(u, \nabla u)=\sum_{*}\left(\frac{\partial}{\partial u}\right)^{l_{0}}\left(\frac{\partial}{\partial \bar{u}}\right)^{\bar{l}_{0}}\left(\frac{\partial}{\partial q}\right)^{l}\left(\frac{\partial}{\partial \bar{q}}\right)^{\bar{l}} F(u, q) \\
& \times \prod_{\beta_{0} \leqslant \beta}\left(J^{\beta_{0}} u\right)^{m\left(\beta_{0}\right)} \times \prod_{j=1}^{N} \prod_{\beta, \leqslant \beta}\left(J^{\beta,} \partial_{x j} u\right)^{m\left(\beta_{j}\right)}
\end{aligned}
$$

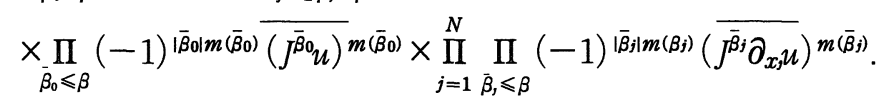

Before the end of this section, we give some embedding lemma which gains time decay.

Lemma 3.4. Let $m$ be an integer satisfying $[m / 2] \geqslant N+2$. Then there exists a constant $C>0$ such that

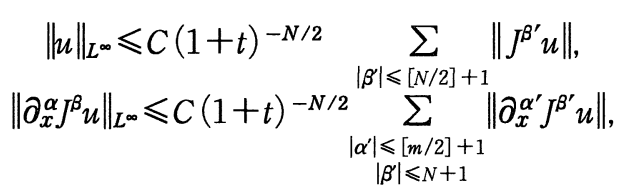

for any $u \in \&\left(\mathbf{R}^{N}\right)$ and for any $\alpha, \beta \in\left(\mathbf{Z}_{+}\right)^{N}$ with $|\alpha| \leqslant[m / 2]+1,|\beta| \leqslant[N / 2]$. (Note $m \geqslant[m / 2]+N+2$.)

Proof. We have only to prove (3.10) because replacing $u$ by $\partial_{x}^{\alpha} J^{\beta} u$ in (3.10) and using (1.8), we can get (3.11). (3.10) is essentially a consequence of Gagliardo-Nirenberg inequality

$$
\|u\|_{L^{\infty}} \leqslant C \sum_{\left|\alpha^{\prime}\right|=n}\left\|\partial_{x}^{\alpha^{\prime}} u\right\|^{N / 2 n}\|u\|^{1-N / 2 n}, \quad \text { for } \quad u \in \mathscr{S}\left(\mathbf{R}^{N}\right), n(\in \mathbf{N}) \geqslant N / 2
$$

Put $\theta=|x|^{2} / 4(1+t)$. We have

$$
\begin{aligned}
\|u\|_{L^{\infty}} & =\left\|e^{-i \theta} u\right\|_{L^{\infty}}, \\
& \leqslant C \sum_{\left|\alpha^{\prime}\right|=n}\left\|\partial_{x}^{\alpha^{\prime}}\left(e^{-i \theta} u\right)\right\|^{N / 2 n} \|_{e^{-i \theta} u \|^{1-N / 2 n}} \\
& =C \sum_{\left|\alpha^{\prime}\right|=n}\left\|e^{i \theta} \partial_{x}^{\alpha^{\prime}}\left(e^{-i \theta} u\right)\right\|^{N / 2 n}\|u\|^{1-N / 2 n} \\
& \leqslant C(1+t)^{-N / 2} \sum_{\left|\alpha^{\prime}\right|=n}\left\|J^{\alpha^{\prime}} u\right\|^{N / 2 n}\|u\|^{1-N / 2 n} \\
& \leqslant C(1+t)^{-N / 2} \sum_{\left|\beta^{\prime}\right| \leqslant n}\left\|J^{\beta^{\prime}} u\right\| .
\end{aligned}
$$

Taking $n=[N / 2]+1(>N / 2)$, we obtain (3.10). 


\section{§4. Proof of Theorem 1.1}

Finally we will complete the proof of Theorem 1.1 . We have only to get $a$ priori estimates in $\cap_{j=0}^{N+1} H^{m-j, j}$ from a viewpoint of Proposition 3.1 and Lemma 3.2 .

Let $\alpha, \beta \in\left(\mathbf{Z}_{+}\right)^{N}$ satisfying $|\alpha|+|\beta| \leqslant m,|\beta| \leqslant N+1$. Using (1.8) and (3.6), we have

$$
\begin{gathered}
\left.\partial_{t}\left(\partial_{x}^{\alpha} J^{\beta} u\right)-i \Delta\left(\partial_{x}^{\alpha} J^{\beta} u\right)-i \sum_{j=1}^{N} \frac{\partial F}{\partial q_{j}} D_{x},\left(\partial_{x}^{\alpha} J^{\beta} u\right)-i(-1)^{|\beta|} \sum_{j=1}^{N} \frac{\partial F}{\partial \bar{q}_{j}} D_{x}, \overline{\left(\partial_{x}^{\alpha} J^{\beta} u\right.}\right)=G(u), \\
G_{\alpha \beta}(u)=\partial_{x}^{\alpha} J^{\beta} F(u, \nabla u)-\sum_{j=1}^{N} \frac{\partial F}{\partial q_{j}} \partial_{x},\left(\partial_{x}^{\alpha} J^{\beta} u\right)-(-1)^{|\beta|} \sum_{j=1}^{N} \frac{\partial F}{\partial \bar{q}_{j}} \partial_{x}\left(\overline{\partial_{x}^{\alpha} J^{\beta} u}\right)
\end{gathered}
$$

By putting $v_{\alpha \beta}={ }^{t}\left(\partial_{x}^{\alpha} J^{\beta} u, \overline{\partial_{x}^{\alpha} J^{\beta} u}\right)$ and $f_{\alpha \beta}(u)={ }^{t}\left(G_{\alpha \beta}(u), \overline{G_{\alpha \beta}(u)}\right),(4.1)$ becomes

$$
\left(\partial_{t}+i\left(A+B_{\beta}(t)\right)\right) v_{\alpha \beta}=f_{\alpha \beta}(u),
$$

where

$$
b_{\beta}(t, x, \xi ; u)=\left[\begin{array}{cc}
-\sum_{j=1}^{N} \frac{\partial F}{\partial q_{j}}(u, \nabla u) \xi_{j} & -(-1)^{|\beta|} \sum_{j=1}^{N} \frac{\partial F}{\partial \bar{q}_{j}}(u, \nabla u) \xi_{j} \\
(-1)^{|\beta|} \sum_{j=1}^{N} \overline{\frac{\partial F}{\partial \bar{q}_{j}}(u, \nabla u) \xi_{j}} & \sum_{j=1}^{N} \overline{\frac{\partial F}{\partial q_{j}}(u, \nabla u) \xi_{j}}
\end{array}\right]
$$

We will apply the method used in $\S 2$ to $(4.2)$.

Concerning the diagonalization, we define

$$
\tilde{\lambda}_{\beta}(t, x, \xi ; u)=
$$

$$
\left[\begin{array}{cc}
0 & -(-1)^{|\beta|} \frac{1}{2} \sum_{i=1}^{N} \frac{\partial F}{\partial \bar{q}_{j}}(u, \nabla u) \xi_{j}\langle\xi\rangle-2 \\
-(-1)^{|\beta|} \frac{1}{2} \sum_{j=1}^{N} \frac{\partial F}{\partial \bar{q}_{j}}(u, \nabla u) \xi_{j}\langle\xi\rangle^{-2} & 0
\end{array}\right],
$$

$\lambda_{\beta}(t, x, \xi ; u)=I+\tilde{\lambda}_{\beta}(t, x, \xi ; u), \quad \lambda_{\beta}^{\prime}(t, x, \lambda ; u)=I-\tilde{\lambda}_{\beta}(t, x, \xi ; u)$.

Next we consider the transformation which eliminates the loss of derivatives. We define

$$
M_{1}(T)=\sup _{t \in[0, T]} \sum_{\substack{|\alpha|+|\beta| \leqslant m-1 \\|\beta| \leqslant N+1}}\left\|\partial_{x}^{\alpha} J^{\beta} u(t)\right\| .
$$


Take $0<\delta<1$. If $M_{1}(T) \leqslant R<\infty$, then there exists a constant $C_{R}>0$ such that

$$
\begin{aligned}
\left|\left(\operatorname{Im} \frac{\partial F}{\partial q_{j}}(u, \nabla u)\right)_{j=1, \ldots, N}\right| & \leqslant C_{R}(|u|+|\nabla u|)^{2} \\
& \leqslant C_{R}\|u\|_{W^{1, \infty}}^{1-\delta}(\langle x\rangle|u|+\langle x\rangle|\nabla u|)^{1+\delta}\langle x\rangle^{-(1+\delta)} .
\end{aligned}
$$

And we have

$$
\begin{aligned}
\langle x\rangle|u|+\langle x\rangle|\nabla u| & \leqslant C(|u|+|\nabla u|)+C(|x u|+|x \nabla u|) \\
& \leqslant C(|u|+|\nabla u|)+C(|J u|+|J \nabla u|)+C(1+t)\left(|\nabla u|+\left|\nabla^{2} u\right|\right) \\
& \leqslant C(1+t)\left(\|u\|_{W^{2, \infty}}+\|J u\|_{W^{1, \infty}}\right) \quad(\text { by }(1.8)) .
\end{aligned}
$$

Here

$$
W^{k, \infty}=W^{k, \infty}\left(\mathbb{R}^{N}\right) \equiv\left\{u \in L^{\infty}\left(\mathbb{R}^{N}\right) \mid\|u\|_{W^{k, \infty}} \equiv \sum_{|\alpha| \leqslant k}\left\|\partial_{x}^{\alpha} u\right\|_{L^{\infty}}<+\infty\right\}
$$

Substituting (4.4) into (4.3) and using (3.10), we have

$$
\begin{aligned}
\left|\left(\operatorname{Im} \frac{\partial F}{\partial q_{j}}(u, \nabla u)\right)_{j=1, \ldots, N}\right| & \leqslant C_{R}(1+t)^{1+\delta}\left(\|u\|_{W^{2, \infty}}+\|J u\|_{W^{1, \infty}}\right)^{2}\langle x\rangle^{-(1+\delta)} \\
& \leqslant C_{R}(1+t)^{-N+1+\delta} M_{1}^{2}(T)\langle x\rangle^{-(1+\delta)}, \text { for } t \in[0, T]
\end{aligned}
$$

Hence we put

$$
\phi(t)=C_{R}(1+t)^{-N+1+\delta} M_{1}^{2}(T), \quad \gamma(s)=\left(1+s^{2}\right)^{-(1+\delta) / 2},
$$

and we define $k(t, x, \xi)$ as in $\S 2$.

Moreover we continue the preparation for a priori estimates. Let

$$
N_{1}(u(t))=\sum_{\substack{|\alpha|+|\beta|=m \\|\beta| \leqslant N+1}}\left\|K(t) \Lambda_{\beta}(t) v_{\alpha \beta}\right\|, \quad N(u(t))=N_{1}(u(t))+\sum_{\substack{|\alpha|+|\beta|=m-1 \\|\beta| \leqslant N+1}}\left\|\partial_{x}^{\alpha} J^{\beta} u(t)\right\| .
$$

We define $M(T)=\sup _{t \in[0, T]} N(u(t))$. Let $l$ be the same integer as in $\S 2$. We take $m$ satisfying $m>N / 2+l+4$ in order that $H^{m-1} \hookrightarrow \mathscr{B}^{l+3}$. If $M_{1}(T) \leqslant R$ and if $u$ is a solution to $(1.1)-(1.2)$, then

$$
\begin{gathered}
C_{B}(t) \leqslant C_{R}\left(\|u(t)\|_{\mathscr{B}^{l+1}}^{2}+\left\|\partial_{t} u(t)\right\|_{\mathscr{B}^{l+1}}^{2}\right) \leqslant C_{R}\|u(t)\|_{\mathscr{P}^{\prime+3}}^{2} \leqslant C_{R}(1+t)^{-N} M_{1}^{2}(T), \\
C_{K}(t) \leqslant C_{R}, \quad C_{r} \leqslant C, \quad C_{2}(t) \leqslant C_{R}\left(1+M_{1}^{5}(T)\right), \\
\sum_{\substack{|\alpha|+|\beta| \leqslant m \\
|\beta| \leqslant N+1}}\left\|\partial_{x}^{\alpha} J^{\beta} u(t)\right\| \leqslant C_{2}^{2}(t) N(u(t)) \leqslant C_{2}^{3}(t) \sum_{\substack{|\alpha|+|\beta| \leqslant m \\
|\beta| \leqslant N+1}}\left\|\partial_{x}^{\alpha} J^{\beta} u(t)\right\|, \quad \text { for } t \in[0, T] .
\end{gathered}
$$


Using (3.11), we have

$$
\begin{aligned}
\left\|f_{\alpha \beta}(u)\right\| & =\sqrt{2}\left\|G_{\alpha \beta}(u)\right\| \\
& \leqslant C_{R}\left(\sum_{\substack{\alpha^{\prime}|+| \beta^{\prime}|\leqslant[m / 2]+1\\
| \beta^{\prime} \mid \leqslant[N / 2]}}\left\|\partial_{x}^{\alpha^{\prime}} J^{\beta^{\prime}} u(t)\right\|\right)^{2} \sum_{\substack{\left|\alpha^{\prime}\right|+\left|\beta^{\prime}\right| \leqslant m \\
\left|\beta^{\prime}\right| \leqslant N+1}}\left\|\partial_{x}^{\alpha^{\prime} J^{\prime}} u(t)\right\| \\
& \leqslant C_{R}(1+t)^{-N}\left(\sum_{\substack{\alpha^{\prime}|+| \beta^{\prime}|\leqslant m\\
| \beta^{\prime} \mid \leqslant N+1}}\left\|\partial_{x}^{\alpha^{\prime}} J^{\beta^{\prime}} u(t)\right\|\right)^{3} \\
& \leqslant C_{R} C_{2}^{6}(t)(1+t)^{-N} M^{2}(T) N(u(t)) .
\end{aligned}
$$

Finally we will carry out a priori estimates. Similarly to $(2.18)-(2.19)$, we obtain

$$
\begin{aligned}
\frac{d}{d t} N(u(t)) & \leqslant C_{R} C_{2}^{7}(t)(1+t)^{-N+1+\delta} M^{2}(T) N(u(t)) \\
& \leqslant C_{R} M^{2}(T)\left(1+M^{35}(T)\right)(1+t)^{-N+1+\delta} N(u(t))
\end{aligned}
$$

Note $N-1-\delta>1$ because $N \geqslant 3$. Gronwall's inequality yields

$$
N(u(t)) \leqslant C_{2}(0) \varepsilon \exp \left(C_{R} M^{2}(T)\left(1+M^{35}(T)\right)\right) .
$$

Since the size of initial data is sufficiently small, then there exists a constant $C_{3}$ which is independent of $\varepsilon$, such that we can replace $C_{2}(0)$ by $C_{3}$. Taking $\sup _{t \in[0, T]}$, we get

$$
M(T) \leqslant C_{3} \varepsilon \exp \left(C_{R} M^{2}(T)\left(1+M^{35}(T)\right)\right)
$$

Since $M(T)$ is continuous with respect to $T, \sup _{T>0} M(T)<\infty$ holds provided that $\varepsilon$ is sufficiently small.

Remark 4.1. Let us consider the case of $N=2, p_{w}=3$. Under the condition (1.5) or (1.6), the global existence results hold as we mentioned in $\S 1$. On the other hand, since our method gives the loss of time-decay rate $1+\delta$, generally we need to assume $p_{w} \geqslant 5$ or another additional conditions, in order to get the global existence results. We will explain this additional condition. We decompose

$$
F(u, q)=F_{3}(u, q)+F_{5}(u, q)
$$

where $F_{3}(u, q)$ is a cubic homogeneous polynomial and $F_{5}(u, q)$ is a higher order 
term. Assume that $\operatorname{Im}\left(\partial F_{3} / \partial q_{j}\right), j=1,2$ satisfies null gauge condition of degree 2 (see Y. Tsutsumi [17]). Namely there exist constants $a_{j k} \in \mathbf{R}, j, k=1,2$ such that

$$
\operatorname{Im} \frac{\partial F_{3}}{\partial q_{j}}(u, \nabla u)=\sum_{k=1,2} a_{j k} \partial_{x_{k}}\left(|u|^{2}\right)=\frac{1}{2 i(1+t)} \sum_{k=1,2} a_{j k}\left(J_{k} u \bar{u}-u \overline{J_{k} u}\right)
$$

In this case, the loss of decay rate in $F_{3}$ is $\delta$ and decay rate in $F_{5}$ is $-3+\delta$. Then we can also prove the global existence because the decay rate is $-2+\delta$ $(<-1)$. For example

$$
F(u, q)=i q_{1} q_{2} \bar{u} \quad\left(\text { i.e. } \quad F(u, \nabla u)=i\left(\partial_{x_{1}} u\right)\left(\partial_{x_{2}} u\right) \bar{u}\right)
$$

satisfies (1.3), (1.4) with $p_{w}=3,(1.7)$ and (4.5). But it does not satisfy (1.6) and then it cannot be treated by gauge transformation.

\section{Acknowledgments}

The author would like to express his sincere gratitude to Professors Y. Ohya and S. Tarama for their valuable advice and constant encouragement. The author also thanks Dr. S. Katayama for his useful comments and conversations.

\section{References}

[1] Chihara. H., Local existence for the semilinear Schrödinger equations in one space dimension, J. Math. Kyoto Univ., 34 (1994), 353-367.

[2] - Local existence for semilinear Schrödinger equations, Math. Japon., 42 (1995), 353367.

[3] Doi, S., On the Cauchy problem for Schrödinger type equations and the regularity of the solutions, J. Math. Kyoto Univ., 34 (1994), 319-328.

[4] Hayashi, N., Global existence of small solutions to quadratic nonlinear Schrödinger equations, Comm. P. D. E., 18 (1993), 1109-1124.

[5] Hayashi, N., and Ozawa, T., Remarks on nonlinear Schrodinger equations in one space dimension, Diff. Integral Eqs., 7 (1994), 453-461.

[6] Global, small radially symmetric solutions to nonlinear Schrödinger equations and a gauge transformation, Diff. Integral Eqs., 8 (1995), 1061-1072.

[ 7 ] Hörmander, L., The analysis of linear partial differential operators III, Springer-Verlag, Berlin. 1985.

[8] Katayama, S., and Tsutsumi, Y., Global existence of solutions for nonlinear Schrödinger equations in one space dimension, Comm. P. D. E., 19 (1994), 1971-1997.

[9] Kenig, C. E., Ponce, G., and Vega, L., Small solutions to nonlinear Schrödinger equations, Ann. I. H. P. Anal. Non Lin., 10 (1993). 255-288. 
[10] Klainerman, S., and Ponce, G., Global, small amplitude solutions to nonlinear evolution equations, Comm. Pure Appl. Math., 36 (1983), 133-141.

[11] Kumano-go, H., Pseudo-differential operators, MIT press, Cambridge, 1981.

[12] Mizohata, S., On the Cauchy problem, Academic Press, New York, 1985.

[13] Ozawa, T., Remarks on quadratic nonlinear Schrödinger equations, Funkcial. Ekvac., 38 (1995), 217-232.

[14] Shatah, J., Global existence of small solutions to nonlinear evolution equations, J. Diff. Eqs., 46 (1982), 409-425.

[15] Soyeur, A., The Cauchy problem for the lshimori equations, J. Funct. Anal., 105 (1992), 233-255.

[16] Takeuchi, J.. On the Cauchy problem for some non-kowalewskian equations with distinct characteristic roots, J. Math. Kyoto Univ., 20 (1980), 105-124.

[17] Tsutsumi, Y., The null gauge condition and the one dimensional nonlinear Schrödinger equation with cubic nonlinearity, Indiana Univ. Math. J., 43 (1994), 241-254. 
